\title{
$92-21476$
}

\section{MODELING THE PILOT IN VISUALLY CONTROLLED FLIGHT}

\author{
Walter W. Johnson \\ NASA Ames Research Center \\ Moffett Field, California \\ and \\ Anil V. Phatak \\ Analytic Mechanics Associates \\ Sunnyvale, Califomia
}

\section{INTRODUCTION}

Numerous experiments have been performed to determine the transfer function for human operators in simple instrument-based feedback control tasks. For example, the simplest model for a human operator is a gain with a time delay, (which usually ranges between 0.15 and 0.4 seconds). However, there have been no comprehensive studies evaluating human control strategies in visually controlled flight (i.e. flight using a visual scene and not instruments.) This paper describes the results of preliminary studies on this topic.

Human visually guided flight control is important both in low level flight, where it predominates, and in higher altitude flights, where instrument failure is always a potential danger. Researchers have applied two general approaches to this problem, one founded in high order perceptual psychophysics, and the other in control systems engineering. These are described below.

\section{PSYCHOPHYSICAL APPROACH}

The psychophysical approach examines what complex optical or perspective relationships people use in self-movement perception, and their sensitivity to such variables. The visual scene is a segment of an optic array, which, in turn, is the two-dimensional perspective mapping of the threedimensional world onto an observation point. This visual scene may be characterized as an array of varying intensity or brightness levels rich in relationships which inform the observer about his orientation and movement (e.g., see [1] for a discussion of some of the cues that are available in a visual flight task). Humans not only can perceptually identify and extract basic optical features such as points and edges, but, they also can directly extract and regulate significant higher-order features such as optical texture size, optical shapes, and spatio-temporal patterns. According to Gibson [2], the optic array contains important features or cues that are directly regulated or controlled during flight. Furthermore, these cues may be related to aircraft state variables in only complex and indirect ways. However, little is known about how humans use these cues for vehicular control.

Unfortunately, it is unclear how well this approach accounts for manually-controlled flight, since perceptual psychologists have typically left the study of active control to the engineering 
community. Furthermore, the psychophysical approach is in direct contrast with the assumption, embodied in many engineering approaches, that pilots operate upon a recovered representation of aircraft states and environmental disturbances, not upon the raw perception. Instead, engineering approaches assume that humans rely on optical variables to retrieve estimates of these state variables, which are, in turn, used for control. Thus the engineering approach has produced control laws which do not reflect control activity that is guided directly by optical variables or patterns.

\section{ENGINEERING APPROACH}

An examination of engineering approaches for analyzing visually- controlled flight reveals two significant threads. One is the use of classical control methodology to describe simply the input/ output behavior of control systems. This thread relies minimally on psychological assumptions and is represented best by the classical input/output quasi-linear describing function representation or model [3]. The other thread is the use of substantial theoretical assumptions about human behavior, in combination with modern control theoretic techniques, to construct models. This thread is represented best by the optimal control model, which is based on a linear, quadratic, gaussian (LQG) optimal control formulation [4]. The describing function approach treats human control as a "black box" problem, and concentrates on measuring and representing input/output relationships. In contrast, the optimal control model formulation encompasses a psychological model which decomposes human control strategy into two cascaded processes operating on the raw input variables.

The optimal control model assumes that humans first process raw perceptual input through a Kalman filter which yields estimates of vehicle and disturbance states. This model also assumes that humans have internal models of the vehicle dynamics and the disturbance inputs that can be represented mathematically in a common, earth-fixed inertial frame of reference. The model also assumes that humans operate upon these estimates using an optimal linear quadratic controller. Application of this model to visual control tasks uses image features or optical variables as the input variables, but then gives these to a Kalman filter for estimating the vehicle and disturbance states. It is these estimated states, and not the optical variables, which are then controlled. This is assumed to be accomplished with a linear full state feedback controller designed to minimize a quadratic cost function.

Thus, modern control theory and the psychophysical approach represent directly competing models of the information humans might use to control flight. The optimal control model presumes that a non-optical frame of reference is used by humans. It poses the control problem as being, in part, one of converting raw optical variables to a second, more useful, form. i.e. vehicle state variables described in the inertial frame of reference. The psychophysical point of view described above assumes that no conversion is necessary, and that the human operates within an optically defined frame of reference. As a result, the control problem is one of selecting the most useful optical variables for specific control tasks and no frame-of-reference transformation is necessary. However, the describing function approach is more compatible with psychophysical investigations as it provides a useful tool for evaluating the optical variables that are correlated most highly with control behavior. 


\section{NASA RESEARCH PROGRAM}

At NASA-Ames we have initiated a research program to understand and model how humans control flight through visual cues. One element has focused upon the value of formulating manual flight control as a problem in selecting and directly controlling optical variables. Toward this end, we have begun by examining flight control strategies in a minimally complex simulation of a visual hover task (see Figure 1.) This task (described more fully in two other reports [5], [6]) uses a simplified vehicle model with only three translational degrees of freedom: longitudinal (fore/aft), lateral (left/right), and vertical (up/down). No rotational motions are simulated. The human operator is given control over only vertical velocity, and told to maintain a constant altitude over the simulated ground plane.

The human operator's task is to use control stick motion to maintain a reference altitude over a grid plane in the presence of longitudinal, lateral, and vertical disturbances. Figure 2 shows the geometric pattern that the operator sees through the "windscreen" of the simulator. This represents what a pilot might see looking out of the window of an aircraft. It shows: (1) a set of ground "meridian" lines that are parallel to the forward gaze direction and fan out from the vanishing point on the horizon; and (2) a set of ground "latitude" lines that cross the field of view horizontally. No other information (i.e. flight instruments) is provided. This perspective view of the grid plane provides a host of potentially useful features or cues that relate in some analytical way to vehicle state variables [x (longitudinal), y (lateral), and $h$ (vertical.)]

Three grid-plane patterns were studied: (1) a wire frame made of lines parallel to the forward gaze direction (meridian grid); (2) a wire frame made of lines orthogonal to the forward gaze direction (latitude grid); or (3) a wire frame made of both orthogonal and parallel grids (square grid). In addition a random terrain structure composed of irregular colored polygons was presented. This condition included all of the optical information available in the square grid, but in a stochastic fashion.

Performance was very good and nearly identical for trials with the square and latitude grids and with the terrain structure. Performance was poor with the meridian grid. For the square and latitude grids and the terrain structure, there was power in the stick output (stick motion) associated with the $\mathrm{x}$ disturbances as well as with the $\mathrm{h}$ disturbances; operators selected and regulated some optical variable(s) that produced stick inputs associated with changes in longitudinal craft position $\mathrm{x}$ in addition to craft altitude $h$. In control terminology, the stick motion showed the presence of an (undesirable) crossfeed from the craft's longitudinal motion, suggesting the choice of optical variable(s) that varied both with altitude and longitudinal motion.

An examination of the optical variables present in the three grid conditions revealed several cues which unambiguously relate to vehicle altitude alone (i.e. are invariant over changes in $x$ and $y$ ). The operator could have used any of the following cues, which vary with altitude alone:

Cue (1) The distance between any two points where the meridians intersect the bottom of the window (e.g. distance between A and B in Figure 2) 
Cue (2) The number of image latitude lines on the window between any two window locations (e.g. three between $\mathrm{M}$ and $\mathrm{N}$ in Figure 2).

Cue (3) The number of image meridian intersections with the bottom of the window (e.g. the five intersections in Figure 2).

Since performance was generally better when grids with latitude lines were explicitly (i.e. the square and latitude grids) and implicitly present (the terrain structure), this might suggest that operators tried to focus on Cue 2; this is the only cue that depends solely upon latitude lines. However, the presence of the significant crossfeed of the longitudinal disturbance into control motion suggests that the operators must have used a mixed cue that reflected both vertical and longitudinal motion. One such cue is:

Cue (4) The visual optical depression angle of a ground latitude line below the horizon. (e.g.in Figure 2 this this is the visual angle, alpha, subtended by the distance, D, of the latitude line image below the horizon)

However, this observation is not a sufficient test of whether or not this cue was used for this hover task. One should be able to identify the specific reference depression angle that accounts for the observed time history of the stick motion and the corresponding performance data. Use of a given reference depression angle, alpha, implies that: (1) the describing functions relating altitude (h) and longitudinal position ( $x$ ) to stick motion have the same shape, and (2) the ratio of low frequency $\mathrm{h}$ and $\mathrm{x}$ gains equal the tangent of alpha.

This technique was used to determine alpha and the corresponding describing functions. The stick response of this model closely follows the data. Operator control response and performance can also be described by using an optimal control formulation. Since this is a simple task, the internal model of the optimal control formulation would assume a representation which includes, at least, the two vehicle and two disturbance state variables associated with $\mathrm{x}$ and $\mathrm{h}$. The presence of $\mathrm{x}$ crossfeed in the stick motion can only be accounted for by choosing a cost function that includes both $\mathrm{x}$ and $\mathrm{h}$ in addition to the control stick motion. However, it does not seem reasonable or parsimonious to assume that a person has an independent estimate of $h$ but does not use it affect control.

\section{CONCLUSION}

Our initial results show that the use of control engineering modeling techniques, together with a psychophysical analysis of information in the perspective scene, holds promise for capturing the manual control strategies used during visual flight. It is important that we analyze behavior in this way before concluding that the description of visual flight control will be a simple modification of previous models. It is premature to conclude that, simply because humans can get around in a threedimensional world in a very capable fashion, that they do this by extracting these dimensions and controlling their vehicles with respect to that three-dimensional frame of reference. For the purpose of control they may remain within the optical frame of reference. 


\section{REFERENCES}

1. A.V. Phatak, M.S. Karmali and E.J. Hartzell, "Development of a pilot model for helicopter visual flight task segments," in AIAA Proceedings on Flight Testing to Identify Pilot workload and Pilot Dynamics, Edwards Air Force Base, CA. AFFTC-TR-82-5, Edited by Michael Frazier and Robert B. Crombie, 1982.

2. J.J. Gibson, The Senses Considered as Perceptual Systems, Houghton Mifflin Co., Boston, 1966.

3. D.T. McRuer and H.R. Jex, "A review of quasi-linear pilot models," IEEE Trans. on Human Factors and Electronics, HFE-8, 1967.

4. D.L. Kleinman, S. Baron, and W.H. Levison, "A control theoretic approach to manned-vehicle systems analysis," IEEE Trans. on Auto Control, AC-16, 1971.

5. W.W. Johnson and A.V. Phatak, "Optical variables and control strategy used in a visual hover task," in Proceedings of IEEE Conference on Systems, Man \& Cybernetics, Cambridge, MA: November 1989.

6. W.W. Johnson, C.T. Bennett, K. O'Donnell and A.V. Phatak, "Optical variables useful in the active control of altitude," in Proceedings of the 23rd Annual Conference on Manual Control, Cambridge, MA: June, 1988. 


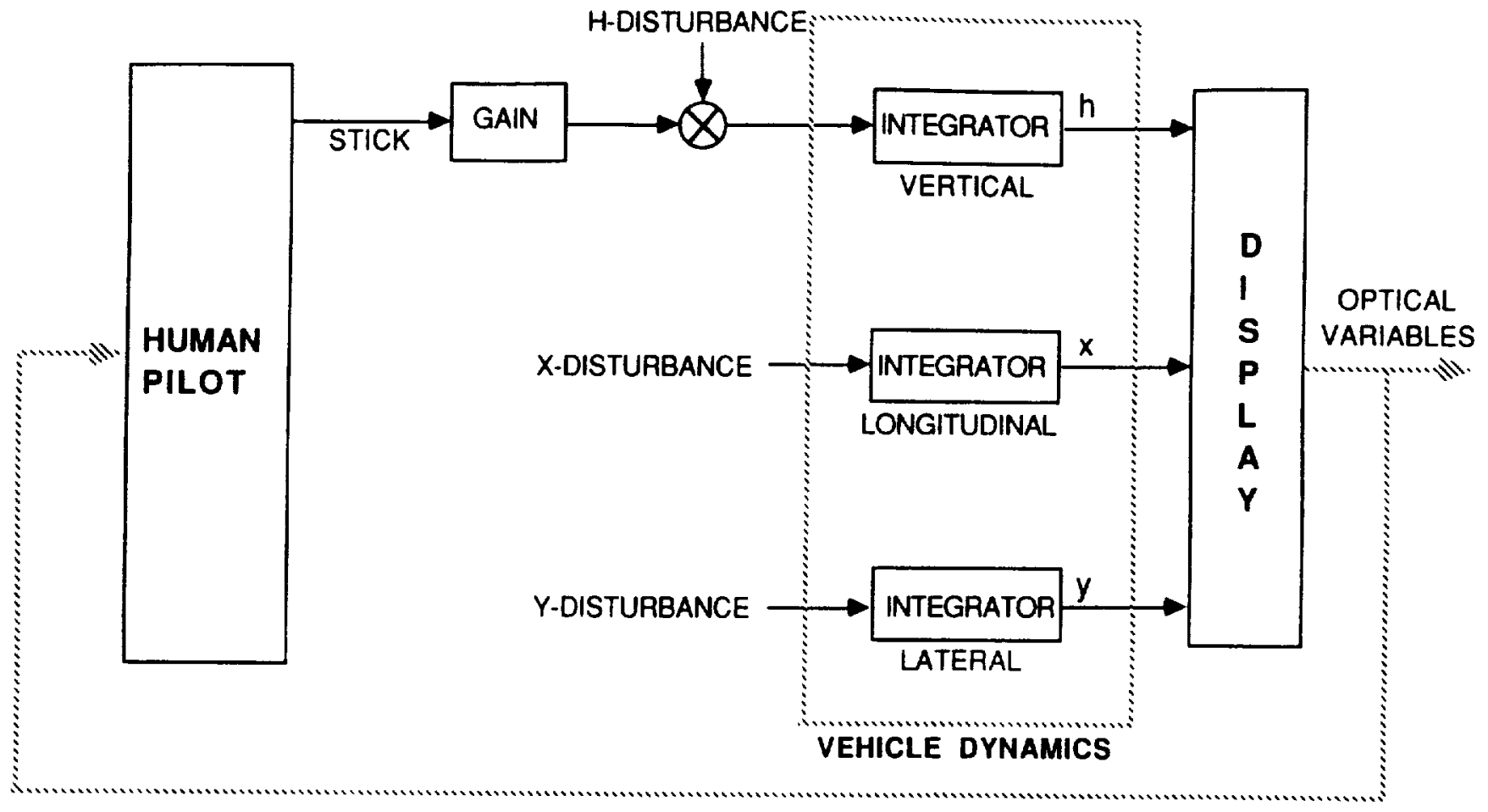

Figure 1. Functional block diagram of visual hover task. 


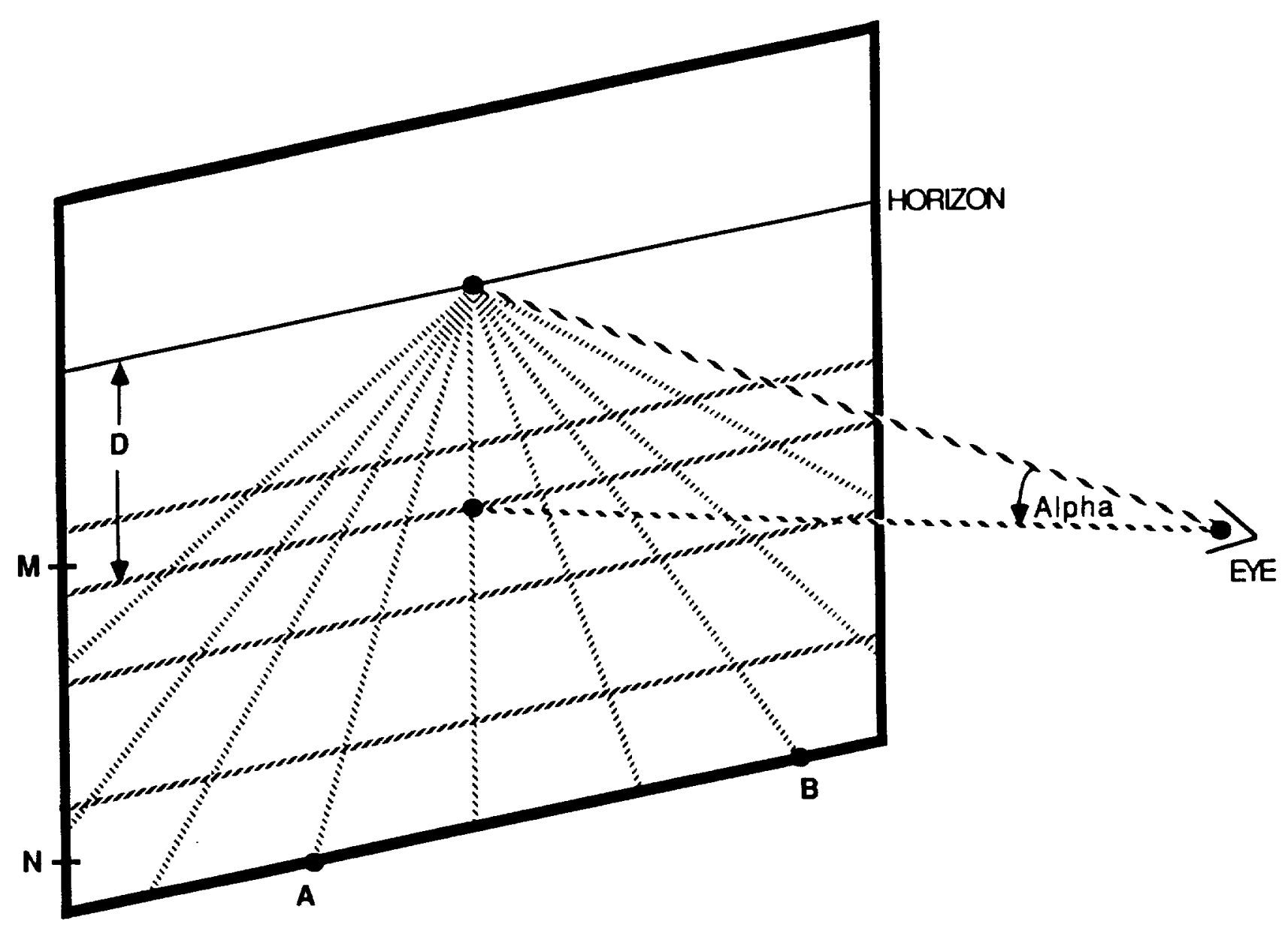

Figure 2. Out-of-the-window view from simulated vehicle cockpit. 
